\title{
Tratamento Coadjuvante da Arterite de Takayasu Através de Angioplastia Transluminal Percutânea com Stents - Relato de Caso e Revisão de Literatura
}

\section{Coadjuvant Treatment of Takayasu Arteritis by Percutaneous Trans/uminal Angioplasty with Stents - Case Report and Literature Review}

\author{
Glauber Tercio de Almeida ${ }^{(1)}$, Bruna Maria Lopes Barbosa ${ }^{(1)}$, Jair Francisco Pestana Biatto ${ }^{(2)}$, \\ Eduardo Casaroto ${ }^{(3)}$, Marco Antônio Araújo da Rocha Loures ${ }^{(4)}$
}

\section{RESUMO}

Os autores fazem uma breve revisão de literatura sobre o uso coadjuvante de stents na arterite de Takayasu e apresentam um relato de caso de uma mulher jovem, 27 anos de idade, com quadro de cefaléia holocraniana acompanhada de náuseas, síncopes e tonturas. Concomitantemente apresentou turvação visual e claudicação de membros superiores. Entre as várias possibilidades, aventou-se a hipótese diagnóstica de arterite de Takayasu, confirmada posteriormente, sendo então realizado tratamento coadjuvante através de angioplastia transluminal percutânea com stents.

Palavras-chave: Takayasu, arterite, angioplastia, stents.

\section{INTRODUÇÃO}

A arterite de Takayasu (AT) é uma vasculite idiopática que acomete preferencialmente aorta toracoabdominal e seus ramos, podendo ainda lesar artérias pulmonares ${ }^{(1)}$. É mais comum em indivíduos de origem asiática, com idade ao diagnóstico entre 10 e 30 anos, principalmente do sexo feminino $(80 \%$ a $90 \%)$. As incidências norte-americana e européia são de 1 a 3 casos/milhão/ano. Tradicionalmente, os glicocorticóides são considerados a pedra fundamental no tratamento medicamentoso da AT, embora agentes citotóxicos, como ciclosporina, metotrexato, ciclofosfamida, micofenolato mofetil têm sido utilizados como drogas poupadoras de esteróides ${ }^{(2)}$.

A seguir o relato de um caso de AT com comprometimento carotídeo e de artérias subclávias bilateralmente, com sintomas neurológicos, cujo tratamento foi a realização

\begin{abstract}
The authors present a brief literature review about coadjuvant treatment with stents in Takayasu Arteritis and report a case of a 27 year old woman showing a holocranial cephalalgia, associated with nausea, fainting and dizziness. Simultaneously she suffered blurred sight and claudication of the upper limbs. Among the different diagnostic possibilities, the hypothesis of Takayasu Arteritis was first considered and later confirmed. Coadjuvant treatment by a percutaneous transluminal angioplasty with stents was performed.
\end{abstract}

Keywords: Takayasu, arteritis, angioplasty, stents.

de angioplastia transluminal percutânea com stents, coadjuvante à terapia com corticosteróides e imunossupressores. $\mathrm{Na}$ seqüência, uma breve revisão de literatura sobre essa abordagem terapêutica na AT.

\section{RELATO DE CASO}

Paciente feminino, 27 anos, encaminhada ao Hospital Universitário Regional de Maringá (HURM) com quadro de cefaléia holocraniana do tipo em aperto, acompanhada de náuseas, vômitos, síncopes e tontura que ocorriam ao se levantar e aos médios esforços. Concomitantemente, apresentou turvação visual, principalmente à direita, e claudicação dos membros superiores. Nega história anterior de febre, artralgia, mialgia e perda de peso. Ao exame físico, encontrava-se em regular estado geral, afebril, descorada, desidratada e havia ausência de pulsos radiais bilateralmente,

Recebido em 16/5/2007. Aprovado, após revisão, em 15/2/2008. Declaramos a inexistência de conflitos de interesse

Hospital Universitário Regional de Maringá (HURM) da Universidade Estadual de Maringá (UEM-PR).

1. Acadêmicos do 6a ano de medicina da UEM-PR

2. Residente (R1) de clínica médica do HURM da UEM-PR

3. Residente (R1) de clínica médica do Hospital Santa Marcelina, São Paulo.

4. Professor auxiliar da disciplina de Reumatologia do Departamento de Medicina da UEM-PR

Endereço para correspondência: Av Cidade Leiria,446,87013-280, Maringá,PR, e-mail: clinicareumatismo@wnet.com.brou marcorl@uol.com.br 
com perfusão periférica diminuída principalmente à direita, sendo impossível a aferição da pressão sanguínea arterial nos membros superiores. Foram realizadas: arteriografias de arco aórtico e carótida, com visível oclusão total das artérias carótida comum direita e subclávia direita, visualizando-se seu terço médio por colaterais (Figura 1). As artérias carótida comum esquerda e subclávia esquerda também se encontravam ocluídas na sua origem (Figura 2). Carótidas interna e externa à esquerda encontravam-se isentas de processo obstrutivo significativo. Ambas as artérias vertebrais são visualizadas por circulação colateral (Figura 1). Com base nos critérios do Colégio Americano de Reumatologia ${ }^{(3)}$, fez-se o diagnóstico de AT, sendo iniciada pulsoterapia com metilprednisolona, $15 \mathrm{mg} / \mathrm{kg} /$ dose, endovenoso por três dias, e mantida posteriormente com prednisolona, $1 \mathrm{mg} /$ $\mathrm{kg} /$ dose. Foi realizada angioplastia de carótida e vertebral com inserção de stent não-medicamentoso, tipo Liberte 5,0 x $24 \mathrm{~mm}$ ao nível da lesão no tronco braquiocefálico, seguido de reexpansão deste com cateter balão Gazelli 6.0/20 (Figuras 3 e 4). A lesão da artéria carótida comum esquerda foi pré-dilatada com cateter balão $4,5 \times 20 \mathrm{~mm}$, e a seguir posicionado e liberado Wallstent 7,0 x $40 \mathrm{~mm}$ (Figura 5). Dessa forma, a angioplastia com implante de stents, para tratamento de lesão obstrutiva no tronco braquiocefálico e na artéria carótida comum esquerda foi realizada com sucesso (Figura 6). A paciente recebeu alta e foi encaminhada ao ambulatório de Reumatologia do HURM em uso de $200 \mathrm{mg}$ de ácido acetilsalicílico, dose única diária, $75 \mathrm{mg}$ de clopidogrel, dose única diária, e metotrexato, $15 \mathrm{mg} /$ semana, não chegando à dose preconizada da última droga em razão da intolerância gástrica. Em evolução posterior, permaneceu assintomática e com a atividade inflamatória da doença sob controle após um ano e seis meses de acompanhamento ambulatorial.

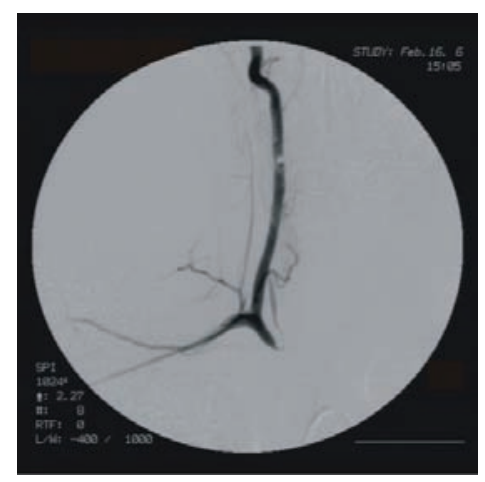

Figura 1 - Arteriografia mostrando obstrução em tronco braquiocefálico.

Rev Bras Reumatol, v. 48, n.2, p. 118-121, mar/abr, 2008

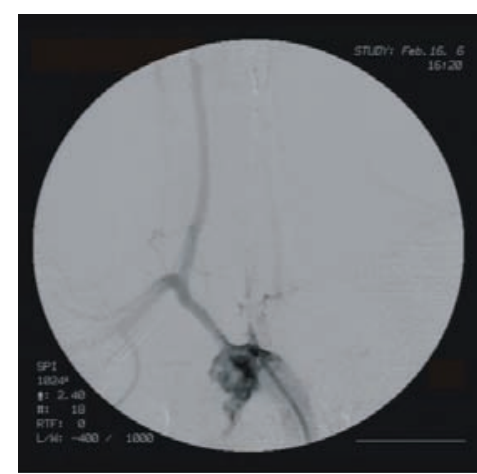

Figura 2 - Arteriografia mostrando obstrução em artéria carótida comum esquerda e subclávia esquerda.

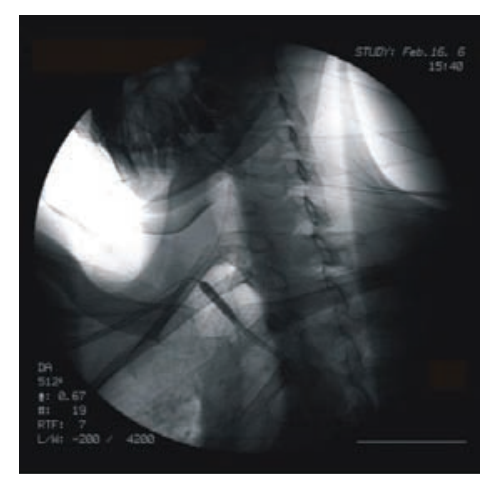

Figura 3 - Angioplastia com balão em tronco braquiocefálico.

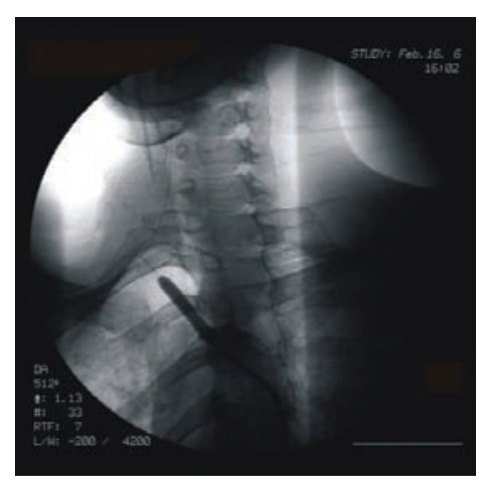

Figura 4 - Implante do stent em tronco braquicefálico.

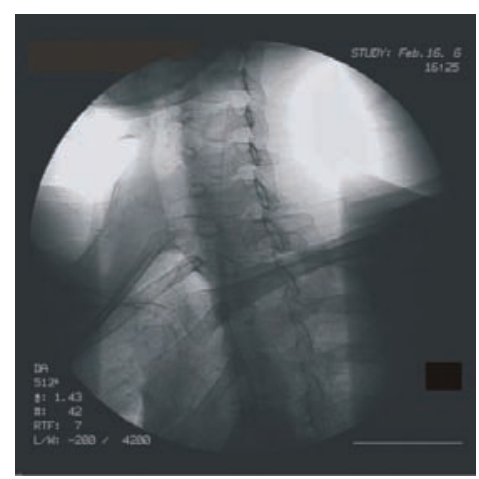

Figura 5 - Angioplastia transcutânea de artéria carótida comum esquerda. 


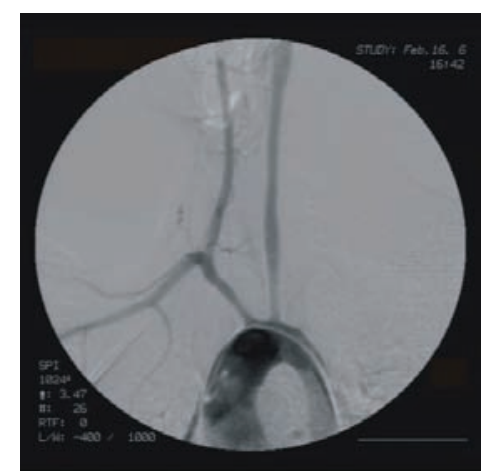

Figura 6 - Resultado final.

\section{DISCUSSÃO}

Com relação à terapêutica intervencionista, a angioplastia com inserção de stents tem se mostrado promissora no tratamento de AT, necessitando ainda de mais estudos com acompanhamento em longo prazo dos pacientes tratados com tais métodos. Entre as indicações para esse procedimento, para controle ou alívio dos sintomas, incluem-se: comprometimento da circulação cervicocraniana (pelo risco de acidente vascular encefálico), hipertensão renovascular por estenose de artéria renal, estenose coronariana significativa (pelo risco de infarto agudo do miocárdio), coarctação aórtica, regurgitação aórtica, claudicação grave em membros, lesões aneurismáticas com risco de ruptura e lesões estenóticas/obstrutivas que determinem efeitos hemodinâmicos ${ }^{(4-6)}$. Em nosso caso, a angioplastia foi indicada devida à possibilidade de isquemia grave em sistema nervoso central, visto que a paciente apresentava sinais e sintomas neurológicos.

Os stents podem ser basicamente classificados em dois tipos: medicamentosos (com liberação local de agentes de ação antiproliferativa como sirolimo e paclitaxel) e não-medicamentosos. Os stents medicamentosos possuem menor taxa de reestenose, porém são mais caros e apresentam maior taxa de trombose local tardia (principalmente, se agentes antiplaquetários são interrompidos $)^{(7)}$. Os stents também podem ser classificados ainda em auto-expansíveis

\section{REFERÊNCIAS}

1. Gotway MB, Araoz PA, Macedo TA, et al.: Imaging findings in Takayasu's arteritis. Am J Roentgenol 184: 1945-50, 2005.

2. Tarrant TK, Allen NB: Other vasculitis syndromes: cryoglobulinemia, Buerger's, Takayasu's, Kawasaki's, and Churg-Strauss. (protótipo = Wallstent) e expansíveis por balão que podem ser filamentares (protótipo = Gianturco-Roubin) ou tubulares $(\text { protótipo }=\text { Palmaz-Schatz })^{(8)}$.

A cirurgia de endarterectomia é aceita como tratamentopadrão para revascularização de lesões ateroscleróticas oclusivas extracranianas, entretanto, em conseqüência da apresentação rara e da fisiopatogenia da AT, não há estudos comparando a endarcterctomia com a implantação de stents em pacientes com $\mathrm{AT}^{(9)}$. As lesões obstrutivas carotídeas, renais e periféricas na AT têm sido tratadas com grande sucesso por meio de angioplastia percutânea com implante de stent, a qual se tem demonstrado segura e com boa relação custo-benefício, sendo alternativa em pacientes com alto risco cirúrgico ${ }^{(10)}$.

Embora a angioplastia transluminal percutânea tenha sido usada na AT, particularmente em lesões focais pequenas, na literatura há descrição apenas de estudos com seguimento a curto prazo após a angioplastia e alguns centros têm descrito a taxa de reestenose de $44 \%$ em 12 meses $^{(4)}$. Outros inconvenientes são embolizações distais ao procedimento. Contudo também é descrito na literatura, principalmente na forma de relatos de caso, que o uso de stents associado a antiagregantes plaquetários próximos a época à realização da angioplastia tem demonstrado resultados mais favoráveis ${ }^{(11,12)}$.

O ideal é que a terapêutica intervencionista seja realizada quando a doença não esteja em atividade. Park et al. ${ }^{(13)}$, em trabalho recente, concluiu que há uma taxa menor de reestenose quando é realizado tratamento imunossupressor pós-intervenção. Geralmente o resultado imediato da angioplastia é bom, sendo que a manutenção da permeabilidade do vaso depende do controle da atividade inflamatória da doença ${ }^{(14)}$. Tal fato ocorreu no caso de nossa paciente, pois depois da intervenção foi mantido o tratamento com metotrexato, mantendo-se a paciente assintomática e com a atividade inflamatória da doença sob controle.

A experiência brasileira em angioplastia transluminal percutânea com stents nos pacientes com AT também tem sido demonstrada por meio de relatos de casos, havendo bons resultados em curto prazo, semelhantemente ao que é relatado na literatura internacional ${ }^{(9)}$.

In: Koopman WJ, Moreland LW: Arthritis and allied conditions: a textbook of rheumatology. $15^{\text {th }} \mathrm{ed}$. Philadelphia: Lippincott Williams \& Wilkins; p 1824-7, 2005.

3. Arend WP, Michel BA, Bloch DA, et al.: The American College of Rheumatology 1990 criteria for the classification of Takayasu arteritis. Arthritis Rheum 33:1129-34, 1990. 
4. Andrews J, Mason JC: Takayasu's arteritis - recent advances in imaging offer promise. Rheumatology 46: 6-15, 2007.

5. Kerr GS, Hallahan CW, Giordano J, et al.: Takayasu arteritis. Ann Intern Med 120: 919-29, 1994.

6. Lupi-Herrera E, Sanchez-Torres G, Marcushamer J: Takayasu's arteritis. Clinical study of 107 cases. Am Heart J 93: 94-103, 1977.

7. Harper RW: Drug-eluting coronary stents - a note of caution. Med J Aust 186: 253-5, 2007.

8. Guérios EE, Bueno RRL, Andrade PMP, Nercolini DC, Pacheco ALA: Stents. Uma revisão da literatura. Arq Bras Cardiol 71: 77-87, 1998 .

9. Tumelero RT, Teixeira JCC, Duda NT, Tognon AP, Rossato M: Tratamento percutâneo multivaso da arterite de Takayasu. Arq Bras Cardiol 87: 234-40, 2006.
10. Sharma BK, Jain S, Bali HK, Jain A, Kumari S: A follow-up study of balloon angioplasty and de-novo stenting in Takayasu arteritis. Int J Cardiol 75(Suppl 1): S147-52, 2000.

11. Takahashi JC, Sakai N, Manaka H, et al.: Multiple supra-aortic stenting for Takayasu arteritis: extensive revascularization and two-year follow-up. Am J Neuroradiol 23: 790-3, 2002.

12. Bali HK, Jain S, Jain A, Sharma BK: Stent supported angioplasty in Takayasu arteritis. Int J Cardiol 66(Suppl 1): S213-20, 1998.

13. Park MC, Lee SW, Park YB, Lee SK, Choi D, Shim WH: Postinterventional immunosuppressive treatment and vascular restenosis in Takayasu's arteritis. Rheumatology 45:600-5, 2006.

14. Bali HK, Bhargava M, Bhatta YK, Sandhu MS: Single stage bilateral common carotid artery stenting in a patient of Takayasu arteritis. Neurol India 49: 87-90, 2001. 